\title{
Spondylodiscitis Associated with Recurrent Serratia Bacteremia Due to a Transjugular Intrahepatic Portosystemic Shunt (TIPS): A Case Report
}

Nuno Marques, Rosa Sá, Filomena Coelho, Saraiva da Cunha and A. Meliço-Silvestre Infectious Diseases Departemtn, University of Coimbra; Coimbra, Portugal

\begin{abstract}
We report a case of spondylodiscitis caused by multiresistant Serratia marcescens in a cirrhotic patient who had several Serratia bacteremias after the placement of a transjugular intrahepatic portosystemic shunt (TIPS) device. We concluded that an endovascular stent that can not be removed makes management of recurrent bacteremia difficult. Furthermore, back pain due to bacteremia is indicative of spondylodiscitis. Serratia marcescens can be an aggressive pathogen, causing spinal infection.
\end{abstract}

Key-Words: Serratia marcescens, recurrent bacteremia, spondylodiscitis, TIPS.

\section{Case Report}

A 59-year-old Caucasian man had liver cirrhosis due to alcohol abuse, which was complicated by portal hypertension and esophageal varices. Because of acute upper gastroenterological bleeding and transesophageal sclerosis of varices, he underwent a transjugular intrahepatic portosystemic shunt (TIPS) procedure performed at our hospital. As he also had aspiration pneumonia, intravenous antibiotic therapy with cefoxitin was done for four days. There was gradual clinical improvement, and he was transferred to his local hospital. However, blood cultures collected seven days after TIPS revealed Serratia marcescens, which was only susceptible to cefotaxime, ceftazidime and imipenem. Imipenem was administered during seven days.

Three weeks after TIPS, the patient developed low-back pain and fever $\left(38.5^{\circ} \mathrm{C}\right)$, without neurological involvement. Lumbar radiography and computed tomography were compatible with degenerative processes. The blood cultures showed S. marcescens, which was susceptible to the previously-mentioned antibiotics. Imipenem was reinitiated for another seven days. Ten weeks after TIPS, he was transferred to our department to determine the source of the bacteremia. On admission, he complained of worsening lowback pain, and he was febrile $\left(38.3^{\circ} \mathrm{C}\right)$. Laboratory blood examination showed C-reactive protein to be $5.7 \mathrm{mg} / \mathrm{dL}$ (normal values: $0.0-0.5 \mathrm{mg} / \mathrm{dL}$ ), erythrocyte sedimentation rate $48 \mathrm{~mm} /$ hr and white blood cell counts $8.8 \times 10^{9} / \mathrm{L}$, with $75 \%$ segmented neutrophils. The blood cultures revealed $S$. marcescens, which was only susceptible to imipenem.

A gallium-67 citrate scan revealed an increased uptake at D9-D11, probably due to spondylodiscitis, which was confirmed by bone scanning with technetium-99m and magnetic resonance imaging. He had no central line devices, and the urine cultures were negative. The abdominal Doppler ultrasound showed a TIPS stent with a normal-direction blood

Received on 1 February 2007; revised 20 August 2007.

Address for correspondence: Dr. Nuno Marques. Serviço de Doenças Infecciosas. Hospitais da Universidade de Coimbra. Praceta Prof. Mota Pinto, 3000-075, Coimbra, Portugal.

The Brazilian Journal of Infectious Diseases 2007;11(5):525-527. (C) 2007 by The Brazilian Journal of Infectious Diseases and Contexto Publishing. All rights reserved. flow. Abdominal ultrasound and chest X-rays, as well as transthoracic echocardiography, were normal.

The patient could only walk with the aid of a thoracolumbar brace, and imipenem was administered from the $10^{\text {th }}$ until the $45^{\text {th }}$ week after TIPS, with substantial clinical improvement and discrete imaging symptom stabilization. His next presentation was 48 weeks after TIPS, with a recrudescence of fever, despite 36 weeks of antibiotic therapy. He had a satisfactory clinical condition, no longer wore a lumbar support, and the lab data (ESR, CRP, WBC) showed a good response. However, $S$. marcescens was again isolated from the blood cultures, and it had the same antibiotic-resistance pattern. Although Doppler ultrasound showed the TIPS device to have no thrombus or vegetations, we considered the hypothesis of TIPS infection or colonization by Serratia sp., and we established a plan to continue anti-Serratia treatment, possibly ab aeterno with imipenem or ertapenem. The patient received antibiotic therapy with imipenem for a further 50 weeks. At that time (98 weeks after TIPS) we decided to suspend antibiotic treatment, as he maintained radiological stabilization of the infectious process. Twenty-six weeks after being discharged without antibiotic therapy, he had no fever or other evidence of bacteremia, and he had no back-pain complaints. The patient was able to go about his daily activities without any disability. Radiography and clinical appearance was suggestive of vertebral bone fusion between D9-D10 and D10-D11. He had no further variceal bleeding.

\section{Discussion}

Serratia marcescens is an aerobic Gram-negative bacillus that belongs to the family Enterobacteriaceae; when it was discovered, in the nineteenth century it was believed to be a microorganism with low virulence [1]. However, since the 1960s, it is considered to be an opportunistic human pathogen. It tends to colonize the respiratory and urinary tracts of adults in health-care institutions and has been associated with outbreaks of nosocomial infections. Among all nosocomial bacteremias, 1.2 to $1.4 \%$ are caused by Serratia species [2-4]. Central nervous infection caused by $S$. marcescens can occur, particularly in neonates with prior sepsis or after a neurosurgical procedure or lumbar puncture [5]. In drug addicts, it can cause endocarditis (often left-sided) and 
osteomyelitis [6,7]. Nevertheless, Serratia rarely affects the bones and joints [8]. Patients with nosocomial infections, diabetes mellitus, renal failure, who have a debilitated immune status, or have traumatic open wounds, as well as the elderly and drug addicts, are the most susceptible to Serratia infections $[8,9]$. These infections are associated with high mortality (25\%-52\%), especially when they progress to bacteremia [9-11].

Serratia spp. can survive under hostile conditions, including in disinfectant solutions, which can become the source for outbreaks; in addition they can spread within or among health care institutions on the hands of staff [1]. The source of most infections of the intervertebral disc is the blood; though it can also occur postoperatively. There are relatively few cases of post-operative spinal infection due to Serratia (two cases after elective lumbar spine surgery, one case after anterior cervical discectomy and fusion and another case after post-lumbar vertebroplasty) $[9,12,13]$. We also found two cases of Serratia osteomyelitis in recently-fractured vertebra [14].

Our patient was profoundly debilitated on admission at our hospital, and he had a respiratory tract infection. Later, he suffered from spondylodiscitis due to hematogenous dissemination of $S$. marcescens following TIPS device placement. Besides hospitalization he had other risk factors for acquiring Serratia infection, including prior antibiotic therapy, chronic-liver disease and an endovascular device. The definition of a TIPS device infection or endotipsitis is controversial; alternatives include sustained bacteremia in a patient with a TIPS device, with or without thrombus, plus either no other identifiable infective focus or an identifiable infective focus that is not considered to be the source of the bacteremia after intense investigation of endotipsitis characterization [15]. Although there was an identifiable infective focus (spondylodiscitis) for the bacteremia, the clinical outcome and the recurrent Serratia bacteremias lead us to believe that the source of the infection was colonization of the TIPS device, even though there were no signs of infection in the Doppler ultrasound exam. If the bacteremias provoke the spondylodiscitis process, we would expect worsening back pain and image symptoms each time they reoccur, which has not always been the case. Recurrent bacteremia is characterized by isolation of the same organism from the blood cultures after at least five days of appropriate antibiotic therapy [4]. Infection of a TIPS devices is rare; the estimated overall infection rate varies from $1.7-5.1 \%$ of the procedures performed; infection can occur several months or years after stent placement $[15,16]$.

Removal of the intravascular device is the best recourse for management of recurrent bacteremias, but this approach is not achievable in some circumstances; this was the case for our patient, because TIPS surgical removal was not possible without liver transplantation. Serratia marcescens is resistant to ampicillin, macrolides and first-generation cephalosporins; treatment usually includes an antipseudomonal beta-lactam and an aminoglycoside [1,17]. Most strains are susceptible to quinolones as well. Monotherapy with a beta-lactam is not advised because resistant mutants can be selected. Furthermore, cross-resistance to aminoglycosides can accompany beta-lactam resistance [4]. Multiresistant strains have been emerging during the last decades; therefore treatment should be based on susceptibility tests. In our case, imipenem was the first treatment choice, and it rapidly became the only option. Cefoxitin is known to be a potent betalactamase inducer; previous utilization can provoke cross resistance [4]. The patient was treated with imipenem for long periods, including 36 continuous weeks, followed by another 50 continuous weeks. Since the resistance pattern of this Serratia infection did not allow oral treatment, our patient had to remain hospitalized during long periods or had to go the hospital daily for intravenous antibiotic therapy.

In one of the largest published series of Serratia bacteremia (122 episodes during a 10-year period), imipenem gave the lowest resistance rate $(0.87 \%)$, followed by amikacin (19.4\%) and cefepime (22.6\%) [4]. Nonetheless, beta-lactamase-mediated carbapenem resistance has been reported for many Gramnegative organisms, including S. marcescens $[18,19]$.

Even though there is no consensus concerning the duration of antibiotic therapy in a spondylodiscitis process, we think that antimicrobial suppression should be continued for a long period.

We do not know if the lengthy antibiotic treatment period was sufficient to resolve the spondylodiscitis process or if it resulted in sterilization of a TIPS-device colonization process. However, we conclude that early diagnosis and aggressive treatment of spondylodiscitis are essential.

\section{References}

1. Eisenstein B.I., Zaleznik D.F. Enterobacteriaceae. In: Mandell G.L., Douglas R.G., Bennett J.E., Dolin E., eds. Principles and Practice of Infectious Diseases, $6^{\text {th }}$. Ed. Philadelphia: Churchill Livingstone, 2005:2579-80.

2. Edmond M.B., Wallace S.E., McClish D.K., et al. Nosocomial bloodstream infections in United States hospitals: a three year analysis. Clin Infect Dis 1999;29:239-44.

3. Gatell J.M., Trilla A., Latorre X., et al. Nosocomial bacteremia in a large Spanish teaching hospital: analysis of factors influencing prognosis. Rev Infect Dis 1988;10:203-10.

4. Choi S.H., Kim Y.S., Chung J.W., et al. Serratia bacteremia in a large university hospital: trends in antibiotic resistance during 10 years and implications for antibiotic use. Infection Control and Hospital Epidemiology 2002;23(12):740-7.

5. Bizzarro M.J., Dembry L.M., Baltimore R.S., Gallagher P.G. Casecontrol analysis of endemic Serratia marcescens bacteremia in a neonatal intensive care unit. Arch Dis Child Fetal Neonatal Ed 2006; [Epub ahead of print].

6. Fishbach R.S., Rosenblatt J.E., Dahlgren J.G. Pyogenic vertebral osteomyelitis in heroin addicts. Calif Med 1973;119:1-4.

7. Mintz L., Mollett G.H. Serratia vertebral osteomyelitis in narcotic addicts [letter]. Ann Intern Med 1975;83:668-9.

8. Svensson O., Parment P.A., Blomgren G. Orthopaedic infections by Serratia marcescens: a report of seven cases. Scand J Infect Dis 1987;19:69-75.

9. Hadjipavlou A.G., Gaitanis I.N., Papadopoulos C.A., Katonis P.G., Kontakis G.M. Serratia spondylodiscitis after elective lumbar spine surgery: a report of two cases. Spine 2002;27(23):E507-12. 
10. Haddy R.I., Mann B.L., Nadkarni D.D., et al. Nosocomial infection in the community hospital: severe infection due to Serratia species. J Fam Pract 1996;42:273-7.

11. Wetanakunakorn C. Serratia marcescens: a review of 44 episodes. Scand J Infect Dis 1989;21:477-83.

12. Kulkarni A.G., Hee H.T. Adjacent level discitis after anterior cervical discectomy and fusion (ACDF): a case report. Eur Spine J 2006;15(suppl.5):S559-63.

13. Olmos M.A., González A.S., Clemente J.D., Tomé C.V. Infected vertebroplasty due to uncommon bactéria solved surgically: a rare and threatening life complication of a common procedure. Spine 2006;31(20):770-3.

14. Lowe J., Kaplan L., Liebergall M., Floman Y. Serratia osteomyelitis causing neurological deterioration after spine fracture. J Bone Joint Surg [Br] 1989;71-B:256-8.
15. Armstrong P.K., MacLeod C. Infection of transjugular intrahepatic portosystemic shunt devices: three cases and review of the literature. Clin Infect Dis 2003;36:407-12.

16. DeSimone J.A., Beavis K.G., Eschelman D.J., Henning K.J. Sustained bacteremia associated with transjugular intrahepatic portosystemic shunt (TIPS). Clin Infect Dis 2000;30:384-6.

17. Ania B.J. Serratia. www.emedicine.com/med/topic2103.htm. Accessed January 29, 2007.

18. Naas T., Vandel L., Sougakoff W., et al. Cloning ans sequence analysis of the gene for a carbapenem-hydrolyzing class A âlactamase, Sme-1, from Serratia marcescens S6. Antimicrob Agents Chemothe 1994;38:1262-70.

19. Cunha B.A. Antibiotic resistance. Med Clin North Am 2000;84:1407-29. 